\title{
A CONTROL-VOLUME FINITE ELEMENT METHOD FOR THE PREDICTION OF THREE-DIMENSIONAL DIFFUSION-TYPE PHENOMENA IN ANISOTROPIC MEDIA
}

\author{
Simon Kattoura, Alexandre Lamoureux, and Bantwal R. (Rabi) Baliga* \\ *Author for correspondence \\ Department of Mechanical Engineering, Heat Transfer Laboratory, McGill University \\ 817 Sherbrooke St. W. \\ Montreal, Quebec H3A 0C3, Canada \\ E-mail: rabi.baliga@mcgill.ca
}

\begin{abstract}
The formulation and testing of a control-volume finite element method (CVFEM) for the prediction of three-dimensional, linear and nonlinear, diffusion-type phenomena in anisotropic media in irregular calculation domains are presented and discussed in this paper. In this CVFEM, the calculation domain is discretized into four-node tetrahedral elements. Contiguous, non-overlapping, polyhedral control volumes are then associated with each node, and the governing differential equation is integrated over these control volumes. The dependent variable is interpolated linearly in each four-node tetrahedral element. Centroidal values of the diffusion coefficients are stored and assumed to prevail over the corresponding tetrahedral element. The source term is linearized, and nodal values of its coefficients are stored and assumed to prevail over the polyhedral sub-control volumes. Using these interpolation functions, the discretized equations, which are algebraic approximations to the integral conservation equations, are derived. The discretized equations, which in general, are nonlinear and coupled, are solved using an iterative procedure. The proposed CVFEM for the solution of anisotropic diffusiontype problems appears to be the first such method that is based on tetrahedral elements and vertex-centered polyhedral control volumes. These features make it particularly attractive for amalgamation with adaptive-grid schemes and applications to problems with complex irregular geometries, such those encountered in the general areas of drying, ground-water flows, conduction in composite materials, injection molding in heterogeneous porous media, and solidification. The proposed three-dimensional CVFEM and its computer implementation were tested using several steady conduction-type problems, for which analytical solutions were constructed using a special technique. In all cases, the agreement between the numerical and analytical solutions was excellent.
\end{abstract}

\section{INTRODUCTION}

Physical phenomena such as heat conduction, potential flow, mass diffusion, flows through porous media in the Darcy regime, electromagnetics and electrostatics, and lubrication flows are governed by equations that have the same general form, akin to that of the classical heat conduction or mass diffusion equations. In this sense, they can all be thought of as diffusion-type phenomena [Patankar (1980)]. Several naturally occurring materials, such as woods and soils, and also engineered materials, such as laminated metal sheets and composites, have properties that are intrinsically dependent on direction. Thus, it is important to account for anisotropy in the modeling of diffusion-type phenomena in such materials.

Elaborating a bit more on the points mentioned above, heat conduction processes in laminated metal sheets, commonly used in the construction of electric power transformers, and also in composites materials, now widely used in the aerospace industry, are anisotropic diffusion-type phenomena [Ozisik (1980); Ozisik and Shouman (1980); Bejan (1984)]. Volume-averaged approaches [Whitaker (1999)] to the modeling of heat and mass transfer in porous media, for example drying of wood or food stuff, ground water flow, and transport of solutes in ground, and modeling of wave propagation in biological tissues, often lead to anisotropic diffusion-type problems [Plumb and Spolek (1985); Ferguson (1995, 1998); Ferguson and Turner (1995, 1996); Kaviany (1995); Harrild and Henriquez (1997); Nield and Bejan (1999); Yang and Lee (1999); Delleur (1999); Lyuksyutov et al. (2001); Schwartz and Zhang (2003)]. Such problems typically involve complex geometries, are three-dimensional, and are governed by equations that cannot be solved analytically. Thus, though examples of analytical approaches to the solution of anisotropic diffusion-type problems are available in the literature [Poon and Chang (1978); Poon (1979); Ozisk (1980); Mikhailov and Ozisik (1981, 1984); Traianno et al. (997)], numerical methods are needed and used for solving the majority of the aforementioned problems.

Detailed descriptions of numerical methods for the solution of fluid flow and heat transfer problems are available in numerous textbooks and handbooks, for example, the works of Patankar (1980), Versteeg and Malalasekera (1995), Zienkiewicz and 
Taylor (2000), Reddy and Gartling (2001), and Minkowycz et al. (2006). There are also many publications on numerical solutions of anisotropic diffusion-type problems. Examples of such publications include the contributions of Perre and Degiovanni (1990), Ozisik (1994), Rabbani (1994), Ferguson (1995, 1998), Ferguson and Turner $(1995,1996)$, Turner and Ferguson (1995a, 1995b), Murthy and Mathur (1998), Perre and Turner (1999), Jayantha and Turner (2003a, 2003b, 2005), and Truscott and Turner (2004). Some probabilistic methods have also been proposed for the solution of anisotropic heat conduction problems [Haji-Sheik and Sparrow (1967); Haji-Sheik (1988)].

The formulation and testing of a control-volume finite element method (CVFEM) for the prediction of three-dimensional, linear and nonlinear, diffusion-type phenomena in anisotropic media in irregular calculation domains are presented and discussed in this paper. CVFEMs combine key concepts from finite volume methods and finite element methods [Baliga and Patankar (1980); Baliga (1997); Baliga and Atabaki (2006)]. Thus, their formulations can be readily interpreted in terms of physically meaningful and relevant quantities such as fluxes, forces, and sources, and, if formulated correctly and consistently, they have the so-called conservative property [Patankar (1980)].

Publications on CVFEMs for the solution of anisotropic diffusion-type problems include the contributions of Ferguson (1995, 1998), Ferguson and Turner (1995, 1996), Turner and Ferguson (1995a, 1995b), Perre and Turner (1999), Jayantha and Turner (2003a, 2003b, 2005), and Truscott and Turner (2004), for example. Other finite-volume-based approaches to the solution of anisotropic diffusion-type problems in complex geometries have also been published, for example, in the works of Murthy and Mathur (1998) and Das et al. (2002). The CVFEM proposed in this paper adds to the repertoire of such methods. It appears to be the first such method in which four-node tetrahedral elements and node-centered (or vertex-based) polyhedral control volumes are used in the formulation. These features make it particularly attractive for the solution of problem with complex geometries, and also future amalgamation with adaptive-grid techniques [Minkowycz et al. (2006)].

\section{NOMENCLATURE}

\begin{tabular}{|c|c|c|}
\hline \multirow{3}{*}{$\begin{array}{l}a_{j}, a_{n}, a^{o}, b \\
A, B, C, D \\
A^{n j}\end{array}$} & & Coefficients in general discretized equation; see Eq. (17) \\
\hline & & Coefficients in interpolation functions \\
\hline & {$\left[\mathrm{m}^{2}\right]$} & $\begin{array}{l}\text { Area of internal quadrilateral surfaces bounding sub- } \\
\text { control-volumes within a tetrahedral element; see } \\
\text { Figures } 1 \text { and } 2 \text { and also Eq. (12) }\end{array}$ \\
\hline $\mathbf{J}$ & {$\left[\mathrm{W} / \mathrm{m}^{2}\right]$} & $\begin{array}{l}\text { General diffusion flux vector; given units apply for heat } \\
\text { conduction problems }\end{array}$ \\
\hline$\overline{\bar{K}}$ & {$[\mathrm{~W} / \mathrm{m} \cdot \mathrm{K}]$} & Thermal conductivity tensor; see Eq. (18), (19), and (24) \\
\hline$L_{x}, L_{y}, L_{z}$ & {$[\mathrm{~m}]$} & $\begin{array}{l}\text { Extents of the calculation domain in the } x, y \text {, and } z \\
\text { directions }\end{array}$ \\
\hline$S, d S$ & {$\left[\mathrm{~m}^{2}\right]$} & Surface area, and surface area element \\
\hline$S_{\phi}$ & {$\left[\mathrm{W} / \mathrm{m}^{3}\right]$} & $\begin{array}{l}\text { Volumetric source term associated with the general } \\
\text { dependent variable } \phi \text {; given units apply for heat }\end{array}$ \\
\hline & & conduction problems \\
\hline$T$ & {$[\mathrm{~s}]$} & Time \\
\hline$T$ & {$\left[{ }^{\circ} \mathrm{C}\right]$} & Temperature \\
\hline$V, d V$ & {$\left[\mathrm{~m}^{3}\right]$} & Volume, and differential volume element \\
\hline$x, y, z$ & {$[\mathrm{~m}]$} & Cartesian coordinates \\
\hline$X, Y, Z$ & {$[\mathrm{~m}]$} & $\begin{array}{l}\text { Cartesian coordinates with origin at the centroid of the } \\
\text { tetrahedral element }\end{array}$ \\
\hline
\end{tabular}

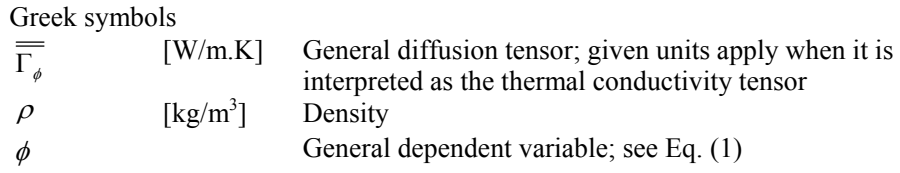

\section{MATHEMATICAL MODEL}

Governing Equations: With reference to the Cartesian $(x, y, z)$ coordinate system, unsteady, three-dimensional, anisotropic, diffusion-type problems are governed by differential equations that can be cast in the following general form [Patankar (1980); Osizik (1980); Kaviany (1995); Nield and Bejan (1999); Oosthuizen and Naylor (1999)]:

$$
\begin{aligned}
& \frac{\partial}{\partial t}(\rho \phi)+\operatorname{div} \mathbf{J}=S_{\phi} \\
& \mathbf{J}=-\overline{\overline{\Gamma_{\phi}}} \bar{\nabla} \phi
\end{aligned}
$$

Here, $\phi$ is a general specific (per unit mass) scalar dependent variable, $\rho$ is the mass density, $\mathbf{J}$ is the diffusion flux vector, $\overline{\overline{\Gamma_{\phi}}}$ is the corresponding second-order tensor of diffusion coefficients, and $S_{\phi}$ is the appropriate volumetric (per unit volume) rate of generation or source term. Casting the governing equations into the form of Eq. (1) allows the development of general-purpose methodology and computer programs that can be used to solve a wide variety of the problems of interest, as was first proposed and pointed out in the seminal works of Spalding [Artemov et al. (2009)]. Each association of $\phi$ with a specific physical dependent variable of relevance to the problem of interest is accompanied by the corresponding specific meanings and expressions (or values) of $\overline{\overline{\Gamma_{\phi}}}$ and $S_{\phi}$ [Patankar (1980)].

It should also be noted that $S_{\phi}$ is meant primarily for modeling actual physical rates of internal generation or source terms. However, it can also be used as a catch-all term [Patankar (1980)]: whatever cannot conveniently be fitted into the other terms in Eqs. (1) and (2) can always be lumped in with $S_{\phi}$.

This tensor, $\overline{\overline{\Gamma_{\phi}}}$, in general, has nine components, $\Gamma_{\phi}^{i j}$, called generalized diffusion coefficients:

$$
\overline{\overline{\Gamma_{\phi}}}=\left[\begin{array}{ccc}
\Gamma_{\phi}^{11} & \Gamma_{\phi}^{12} & \Gamma_{\phi}^{13} \\
\Gamma_{\phi}^{21} & \Gamma_{\phi}^{22} & \Gamma_{\phi}^{23} \\
\Gamma_{\phi}^{31} & \Gamma_{\phi}^{32} & \Gamma_{\phi}^{33}
\end{array}\right]
$$

It should be noted that the generalized diffusion tensor, $\overline{\overline{\Gamma_{\phi}}}$, is symmetric [Osizik (1980)]: thus, $\Gamma_{\phi}^{i j}=\Gamma_{\phi}^{j i}$.

In the case of anisotropic media that are being considered here, the components of the diffusion flux vector along the positive $x, y$, and $z$ directions are given by the following expressions: 
$J_{x}=-\left(\Gamma_{\phi}^{11} \frac{\partial \phi}{\partial x}+\Gamma_{\phi}^{12} \frac{\partial \phi}{\partial y}+\Gamma_{\phi}^{13} \frac{\partial \phi}{\partial z}\right)$

$J_{y}=-\left(\Gamma_{\phi}^{21} \frac{\partial \phi}{\partial x}+\Gamma_{\phi}^{22} \frac{\partial \phi}{\partial y}+\Gamma_{\phi}^{23} \frac{\partial \phi}{\partial z}\right)$

$J_{z}=-\left(\Gamma_{\phi}^{31} \frac{\partial \phi}{\partial x}+\Gamma_{\phi}^{32} \frac{\partial \phi}{\partial y}+\Gamma_{\phi}^{33} \frac{\partial \phi}{\partial z}\right)$

Therefore, for an anisotropic medium, the diffusion flux vector $\mathbf{J}$ at a point on a constant- $\phi$ surface is not necessarily normal to that surface.

Boundary Conditions: The boundary conditions are specific to each problem of interest. Typically, three kinds of boundary conditions are commonly encountered in the modeling of diffusion-type phenomena: 1) specified value of $\phi ; 2)$ specified diffusion flux of $\phi$, normal to the domain boundary, $J_{n}$.; and 3) specification of flux via a reference value of the dependent variable far from the boundary, $\phi_{\text {ref }}$, and a transfer coefficient, $\mathfrak{I}$, such that $J_{n \text {,into domain }}=\mathfrak{I}\left(\phi_{\text {ref }}-\phi_{\text {boundary }}\right)$. Other boundary conditions, if encountered, can always be recast in the form of the aforementioned three conditions. For example, non-linear boundary conditions can be linearized and cast in a form akin to the third type, or a linear combination of all three types.

Initial Conditions: The initial conditions specify the distribution of the dependent variables, $\phi$, at time $t=0$. Again, the initial conditions are problem specific.

\section{FORMULATION OF THE NUMERICAL METHOD}

The proposed method belongs to the family of control-volume finite element methods (CVFEMs). Detailed discussions of CVFEMs are available in the works of Baliga (1997) and Baliga and Atabaki (2006). Thus, only some of the main aspects of the formulation of the proposed CVFEM for the solution of the anisotropic diffusion problems of interest here are succinctly presented in this section.
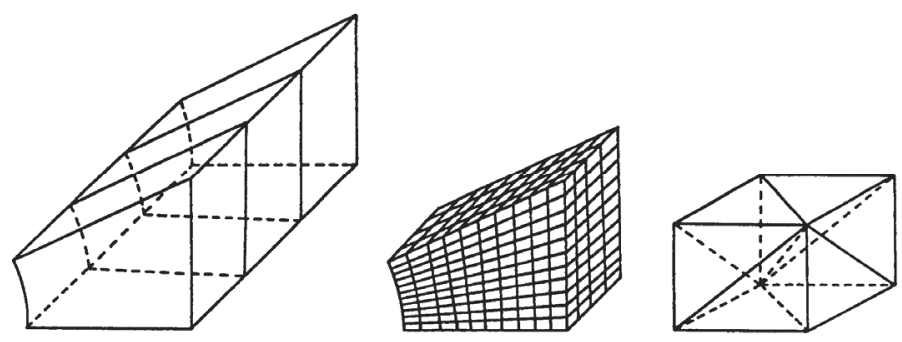

Figure 1 Three-step discretization of a calculation domain into tetrahedral elements: (a) division into slabs; (b) division of the slabs into eight-node brick elements; and (c) subdivision of the eight-node brick elements into four-node tetrahedral element.

Domain Discretization: The calculation domain is divided first into four-node tetrahedral elements; and then each node in the calculation domain is associated with contiguous, nonoverlapping, polyhedral control volumes that fill the calculation domain. The proposed method is designed to work with complex geometries and unstructured grids. Here, however, for ease of presentation, the aforementioned discretization of threedimensional calculation domains into four-node tetrahedral elements is illustrated in Figure 1 with respect to a relatively simple geometry and a three-step procedure. In the proposed CVFEM, the simple four-node tetrahedral element is preferred to brick or parallelepiped elements, because the latter introduce the complication of isoparametric transformations [Strang and Fix (1973); Baliga and Patankar (1980); LeDain-Muir and Baliga (1988)]. In the proposed CVFEM, curved boundary surfaces are approximated by piecewise-plane surfaces, and isoparametric transformations are not required.

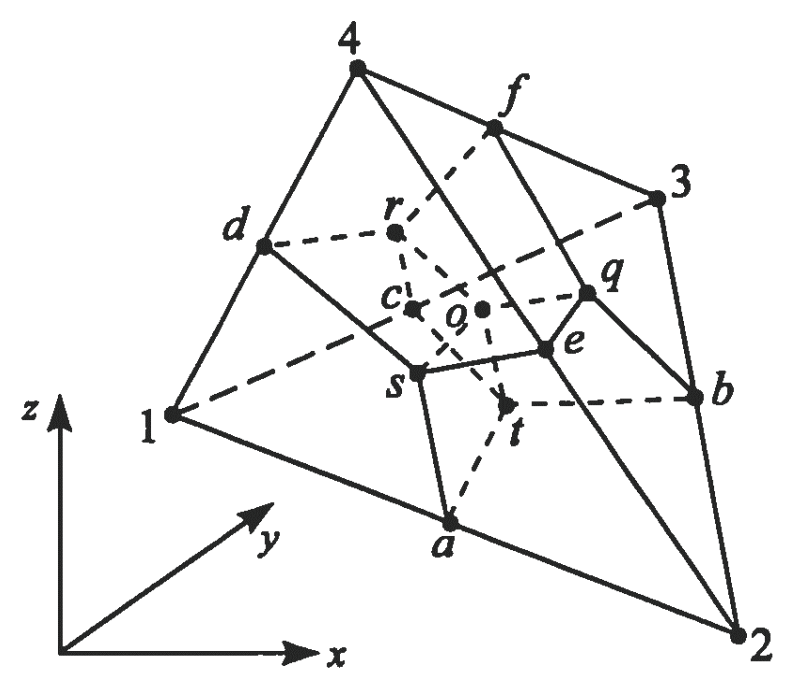

Figure 2 Division of a tetrahedral element into portions of polyhedral control volumes and related nomenclature.

Following the discretization of the calculation domain into four-node tetrahedral elements, each element is divided into four equal volumes, as illustrated in Figure 2. The procedure for this subdivision of the elements into the four equal volumes is the following: the centroid, $o$, of each tetrahedral element is first joined by straight lines to the centroids $q, r, s$, and $t$ of the four triangular surfaces that make up the tetrahedron; then the centroid of each triangular surface is joined by straight lines to the midpoint of the corresponding sides. This procedure generates six plane quadrilateral surfaces within each tetrahedral element that divide it into four equal sub-control-volumes, $V^{l}, V^{2}, V^{3}$, and $V^{4}$, which are not necessarily similarly-shaped. Collectively, these surfaces create polyhedral control volumes associated with each node in the calculation domain and have the following desirable properties: they do not impose any restriction on the size or shape of the tetrahedral elements; they do not overlap; and, collectively, they fill the calculation domain completely.

Integral Conservation Equations: An integral formulation corresponding to Eqs (1) and (2) is obtained by applying the conservation principle for $\phi$ to a control volume, $V$, which is fixed in space: 
$\int_{V} \frac{\partial}{\partial t}(\rho \phi) d V+\int_{\partial V} \mathbf{J} . \mathbf{n} d S=\int_{V} S_{\phi} d V$

Here, $\partial V$ is the surface of the control volume $V$, and $\mathbf{n}$ is a unit outward normal to the differential area element $d S$. The resulting integral conservation equation, when applied to the polyhedral control volume surrounding node 1 of the tetrahedral element shown in Figure 2, can be cast in the following form:

$\left[\begin{array}{l}\int_{\text {latcdsor }} \frac{\partial}{\partial t}(\rho \phi) d V+\int_{\text {atos }} \mathbf{J} . \mathbf{n} d S+\int_{\text {ctor }} \mathbf{J} . \mathbf{n} d S+\int_{\text {latcdsor }} S_{\phi} d V\end{array}\right]+$

$\left[\begin{array}{l}\text { Similar contributions from the other } \\ \text { elements associated with node } 1\end{array}\right]+$

[Boundary contributions, if applicable $]=0$

Interpolation Functions: Interpolation functions for the dependent variable, $\phi$, the diffusion coefficients, $\Gamma_{\phi}^{i j}$, the volumetric source term, $S_{\phi}$, and the mass density, $\rho$, are required for the derivation of algebraic approximations to the integral conservation equations such as Eq. (6).

Within each tetrahedral element, the centroidal values of $\rho$ and $\Gamma_{\phi}^{i j}$ are stored and assumed to prevail over the element. The source term is appropriately linearized, if required, and cast in the form:

$S_{\phi}^{i}=S_{c}^{i}+S_{p}^{i} \phi^{i}$

The coefficients in this equation, $S_{C}^{i}$ and $S_{P}^{i}$, associated with the node $i(i=1,2,3,4)$ are stored and assumed to prevail over the corresponding sub-control-volume, $V^{i}$, contained within the tetrahedral element.

In each four-node tetrahedral element, the scalar dependent variable, $\phi$, is interpolated linearly, in terms of local, centroidal-based, Cartesian coordinates, $X, Y$, and $Z$ :

$$
\begin{aligned}
& \phi=A X+B Y+C Z+D \\
& X=\left(x-x_{o}\right) ; Y=\left(y-y_{o}\right) ; Z=\left(z-z_{o}\right) \\
& x_{o}=\left(\sum_{n=1}^{4} x_{n} / 4\right) ; y_{o}=\left(\sum_{n=1}^{4} y_{n} / 4\right) ; z_{o}=\left(\sum_{n=1}^{4} z_{n} / 4\right)
\end{aligned}
$$

The constants $A, B, C$, and $D$ in this interpolation function can be uniquely determined in terms of the $X, Y$, and $Z$, coordinates of the four nodes of the tetrahedral element and their corresponding values of $\phi$, and cast in the following forms:

$$
\begin{aligned}
& A=\left(A_{1} \phi_{1}+A_{2} \phi_{2}+A_{3} \phi_{3}+A_{4} \phi_{4}\right) / D e t \\
& B=\left(B_{1} \phi_{1}+B_{2} \phi_{2}+B_{3} \phi_{3}+B_{4} \phi_{4}\right) / D e t \\
& C=\left(C_{1} \phi_{1}+C_{2} \phi_{2}+C_{3} \phi_{3}+C_{4} \phi_{4}\right) / D e t \\
& D=\phi_{1}+\left(D_{1} \phi_{1}+D_{2} \phi_{2}+D_{3} \phi_{3}+D_{4} \phi_{4}\right) / D e t
\end{aligned}
$$

In various constant in this equation can be worked out using the following expressions:

$$
\begin{aligned}
X_{i j}= & \left(X_{i}-X_{j}\right) ; Y_{i j}=\left(Y_{i}-Y_{j}\right) ; Z_{i j}=\left(Z_{i}-Z_{j}\right) \\
\text { Det }= & \left\{X_{14}\left(Y_{24} Z_{34}-Y_{34} Z_{24}\right)+Y_{14}\left(X_{34} Z_{24}-X_{24} Z_{34}\right)+\right. \\
& \left.Z_{14}\left(X_{24} Y_{34}-X_{34} Y_{24}\right)\right\} \\
A_{1}= & \left(Y_{24} Z_{34}-Y_{34} Z_{24}\right) ; A_{2}=\left(Y_{34} Z_{14}-Y_{14} Z_{34}\right) \\
A_{3}= & \left(Y_{14} Z_{24}-Y_{24} Z_{14}\right) ; A_{4}=-\left(A_{1}+A_{2}+A_{3}\right) \\
B_{1}= & \left(Z_{24} X_{34}-Z_{34} X_{24}\right) ; B_{2}=\left(Z_{34} X_{14}-Z_{14} X_{34}\right) \\
B_{3}= & \left(Z_{14} X_{24}-Z_{24} X_{14}\right) ; B_{4}=-\left(B_{1}+B_{2}+B_{3}\right) \\
C_{1}= & \left(X_{24} Y_{34}-X_{34} Y_{24}\right) ; C_{2}=\left(X_{34} Y_{14}-X_{14} Y_{34}\right) \\
C_{3}= & \left(X_{14} Y_{24}-X_{24} Y_{14}\right) ; C_{4}=-\left(C_{1}+C_{2}+C_{3}\right) \\
D_{1}= & -\left(A_{1} X_{1}+B_{1} Y_{1}+C_{1} Z_{1}\right) ; D_{2}=-\left(A_{2} X_{1}+B_{2} Y_{1}+C_{2} Z_{1}\right) \\
D_{3}= & -\left(A_{3} X_{1}+B_{3} Y_{1}+C_{3} Z_{1}\right) ; D_{4}=-\left(A_{4} X_{1}+B_{4} Y_{1}+C_{4} Z_{1}\right)
\end{aligned}
$$

Derivation of Discretized Equations: The discretized equations are obtained by first using the above-mentioned interpolation functions to derive algebraic approximations to the element contributions to integral conservation equation, similar to the first set of bracketed terms in Eq. (6), and then assembling these element contributions appropriately [Baliga and Patankar (1980); LeDain-Muir and Baliga (1986); Baliga (1997); Baliga and Atabaki (2006)].

Using Eqs. (4) and (8), the components of the diffusion flux vector, $\mathbf{J}$, can be approximated as follows:

$$
\begin{aligned}
& J_{x}=-\left(A \Gamma_{\phi}^{11}+B \Gamma_{\phi}^{12}+C \Gamma_{\phi}^{13}\right) \\
& J_{y}=-\left(A \Gamma_{\phi}^{21}+B \Gamma_{\phi}^{22}+C \Gamma_{\phi}^{23}\right) \\
& J_{z}=-\left(A \Gamma_{\phi}^{31}+B \Gamma_{\phi}^{32}+C \Gamma_{\phi}^{33}\right)
\end{aligned}
$$

With reference to the tetrahedral element 1234 shown in Figure 2 , and the related notation, let $A^{n j}$ be the area of one of the three interior quadrilateral surfaces bounding the sub-control-volume $V_{j}$ associated with any node $j(n=1,2$, or $3 ; j=1,2,3$, or 4$)$; and let $\mathbf{n}^{n j}$ denote the unit normal to $A^{n}$ pointing out of the subcontrol-volume $V_{j}$. Then the total rate of diffusion transport across surface $A^{n j}$ in the direction of the unit normal $\mathbf{n}^{n j}$ can be approximated as follows:

$$
\int_{A^{n j}} \mathbf{J} . \mathbf{n}^{n j} d S=-A^{n j}\left[\begin{array}{l}
\left(A \Gamma_{\phi}^{11}+B \Gamma_{\phi}^{12}+C \Gamma_{\phi}^{13}\right) n_{1}^{n j}+ \\
\left(A \Gamma_{\phi}^{21}+B \Gamma_{\phi}^{22}+C \Gamma_{\phi}^{23}\right) n_{2}^{n j}+ \\
\left(A \Gamma_{\phi}^{31}+B \Gamma_{\phi}^{32}+C \Gamma_{\phi}^{33}\right) n_{3}^{n j}
\end{array}\right]
$$


Where $n_{1}^{n j}, n_{2}^{n j}$, and $n_{3}^{n j}$ are the components of the unit vector $\mathbf{n}^{n j}$ in the $x, y$, and $z$ directions, respectively. Using the expressions given in Eq. (10), and the expressions for the components of the unit normal $\mathbf{n}^{n j}$, along with the expression in Eq. (12), the algebraic approximations to the rates of diffusion transport across the three interior quadrilateral surfaces bounding the sub-control-volume $V_{j}$ and flowing out of this volume can be cast in the following compact form:

$\int_{A^{n j}} \mathbf{J} . \mathbf{n}^{n j} d S=-\sum_{k=1}^{4}\left(\sum_{l=1}^{3}\left(F_{k}^{l} E_{l}^{n j}\right)\right) \phi_{k}=\sum_{k=1}^{4} C_{k}^{n j} \phi_{k}$

for $k=1,2,3$, and 4 ; and $l=1,2$, and 3 ; and

$F_{k}^{l}=\left(A_{k} \Gamma_{\phi}^{l 1}+B_{k} \Gamma_{\phi}^{l 2}+B_{k} \Gamma_{\phi}^{l 3}\right) /$ Det

$E_{l}^{n j}=A^{n j} n_{l}^{n j}$

$C_{k}^{n j}=-\sum_{l=1}^{3}\left(F_{k}^{l} E_{l}^{n j}\right)$

Using a simple fully-implicit time-integration scheme for the unsteady term in Eq. (6), the following algebraic approximation is obtained for the element contribution to the unsteady term associated with node $j$ :

$\int_{V^{j}} \frac{\partial}{\partial t}(\rho \phi) d V=\frac{V_{e}}{4} \rho \frac{\phi_{j}^{m}-\phi_{j}^{m-1}}{\Delta t}$

In this equation, $V_{e}$ is the volume of the tetrahedral element, and is given by $V_{e}=\operatorname{Det} / 6$. The values of $\phi_{j}$ at time levels $m$ and $(m-1)$ are denoted by $\phi_{j}^{m}$ and $\phi_{j}^{m-1}$, respectively. The volume of any one of the four equal sub-control-volumes within the element is given by $V_{j}=V_{e} / 4$. It should be noted in this context that higher-order time integration scheme could be incorporated relatively easily in this formulation, but that could entail putting some stability limits of the time step [Patankar (1980)].

The element contribution to the source term associated with the node $j$ is approximated as follows:

$$
\int_{V^{j}} S_{\phi} d V=\frac{V_{e}}{4} S_{c}^{j}+\frac{V_{e}}{4} S_{p}^{j} \phi_{j}
$$

Finally, the algebraic approximation to the full element contribution to the integral conservation equation for node 1 can be expressed compactly as follows:

$$
\begin{aligned}
& {\left[\begin{array}{l}
\int_{\text {latcdsor }} \frac{\partial}{\partial t}(\rho \phi) d V+\int_{\text {atos }} \mathbf{J} . \mathbf{n} d S+\int_{\text {ctor }} \mathbf{J . n} d S+ \\
\int_{d s o r} \mathbf{J} . \mathbf{n} d S-\int_{1 \text { atcdsor }} S_{\phi} d V
\end{array}\right]} \\
& =C_{1}^{1} \phi_{1}^{m}+C_{2}^{1} \phi_{2}^{m}+C_{3}^{1} \phi_{3}^{m}+C_{4}^{1} \phi_{4}^{m}+\text { Const }^{1}
\end{aligned}
$$

Similar algebraic approximations can be derived for the element contributions to the integral conservation equations associated with nodes 2,3 , and 4 .

The algebraic approximations to the boundary contributions are derived in a similar manner. Guidance in this regard can be obtained from the derivations presented in Baliga and Patankar (1980), LeDain-Muir and Baliga (1986), and Baliga and Atabaki (2006).

The discretized equations can be cast in the following compact and general form:

$a_{j} \phi_{j}^{m}=\sum_{n} a_{n} \phi_{n}^{m}+a_{j}^{0} \phi_{j}^{m-1}+b_{j}$

Solution of Discretized Equations: The discretized equations derived in the last section form a set of simultaneous algebraic equations that, in general, could be nonlinear. At each time step, the following simple successive-substitution sequential iterative variable adjustment (SIVA) procedure was used to solve these equations:

1. Guess all unknown values of $\phi$ in the calculation domain.

2. Calculate the coefficients in the linearized form of the discretized equations, using the currently available values of the dependent variables.

3. Sequentially solve the resulting sets of linear, or nominally linear, discretized equations.

4. Repeat steps 2 and 3 until convergence.

Step 3 in the above algorithm involves the sequential solution of sets of linear, or nominally linear, algebraic equations. In the proposed method, these equations were solved by a plane-by-plane iterative procedure. When the discretized equations associated with the nodes in any particular plane were being solved, the currently available values of the dependent variables at the nodes on neighboring planes were treated as known values. A line-by-line tri-diagonal-matrixalgorithm (TDMA) was used to solve the discretized equations in the plane of interest. Details of such methods are available in Patankar (1980) and Sebben and Baliga (1995). This procedure was repeated plane-by-plane until the entire calculation domain was covered. Such iterative sweeps, in alternating coordinate directions, were continued until convergence. In this work, the convergence criterion was that the absolute values of suitably normalized residuals of the discretized equations were all less than or equal to $10^{-6}$.

The above-mentioned plane-by-plane-cum-line-by-line method for solving the linearized and decoupled sets of discretized equations is relatively simple and adequate for solving some test problems and also some simple demonstration problems. For practical applications that require large grids, however, incorporation of convergence acceleration techniques, such as algebraic multigrid methods [Briggs (1987)], or some other efficient iterative solution methods such as the bi-conjugate gradient stabilized (BI-CGSTAB) method [Van der Vorst (1992)], is highly desirable and recommended. 


\section{RESULTS AND DISCUSSION}

The testing of the proposed three-dimensional CVFEM and its computer implementation was done by using steady conductiontype problems, for which analytical solutions were constructed using a special technique proposed by Patankar (1980). This technique consists of the following steps: 1) choose a calculation domain, regular or irregular; 2) propose a reasonable distribution of temperature as a solution; 3) prescribe boundary conditions using the values of the prescribed temperature distribution on the boundaries; and 4) substitute the proposed temperature distribution in the governing equation, and determine the volumetric source term that would ensure that the proposed temperature distribution is indeed a solution. This technique works when the solution to the governing equations (with the calculated source term) and the proposed boundary conditions is unique. Once the aforementioned steps are completed, the proposed numerical method is used to solve the problem (compute the temperature) with the prescribed calculation domain, boundary conditions, and the calculated source terms as inputs.

In the proposed test problems, the focus was only on steadystate problems. Thus, the governing equation for the test problems was the following:

$\nabla \cdot(\overline{\bar{K}} \nabla T)+S=0$

In this equation, $\overline{\bar{K}}$ is the conductivity tensor:

$\overline{\bar{K}}=\left[\begin{array}{lll}k^{11} & k^{12} & k^{13} \\ k^{21} & k^{22} & k^{23} \\ k^{13} & k^{23} & k^{33}\end{array}\right]$

In the test problems, the components of the conductivity tensor given in Eq. (19) are assumed to be constant. Thus, with respect to a Cartesian coordinate system, and keeping in mind that the conductivity tensor is symmetric [Ozisik (1980)], Eqs. (18) and (19) can be combined and rewritten as follows:

$$
\begin{aligned}
& k^{11} \frac{\partial^{2} T}{\partial x^{2}}+k^{22} \frac{\partial^{2} T}{\partial y^{2}}+k^{33} \frac{\partial^{2} T}{\partial z^{2}}+ \\
& 2\left(k^{12} \frac{\partial^{2} T}{\partial x \partial y}+k^{13} \frac{\partial^{2} T}{\partial x \partial z}+k^{23} \frac{\partial^{2} T}{\partial y \partial z}\right)+S=0
\end{aligned}
$$

To ensure the testing of all aspects of the proposed CVFEM, the following three different temperature distributions (one linear, and two non-linear) and three different conductivity tensors (isotropic, orthotropic, and anisotropic) were considered:

\section{Linear temperature distribution (LTD)}

$T=A\left(X / L_{x}\right)+B\left(Y / L_{y}\right)+C\left(Z / L_{z}\right)+D$
2. Non-linear temperature distribution, Type 1

$$
T=A\left(1-\frac{X}{L_{x}}\right)^{2}+B\left(1-\frac{Y}{L_{y}}\right)^{2}+C\left(1-\frac{Z}{L_{z}}\right)^{2}
$$

3. Non-linear temperature distribution, Type 2

$$
T=A\left(1-\frac{X}{L_{x}}\right)\left(1-\frac{Y}{L_{y}}\right)\left(1-\frac{Z}{L_{z}}\right)
$$

The conductivity tensors for the isotropic, orthotropic, and anisotropic cases were set to the following values:

$$
\begin{aligned}
& \overline{\bar{K}}_{\text {isot }}=\left(\begin{array}{lll}
1 & 0 & 0 \\
0 & 1 & 0 \\
0 & 0 & 1
\end{array}\right) \\
& \overline{\bar{K}}_{\text {Orthot. }}=\left(\begin{array}{ccc}
1 & 0 & 0 \\
0 & 1.05 & 0 \\
0 & 0 & 1.1
\end{array}\right) \\
& \overline{\bar{K}}_{\text {Anisot. }}=\left(\begin{array}{ccc}
1 & 0.05 & 0.1 \\
0.05 & 1.05 & 0.05 \\
0.1 & 0.05 & 1.01
\end{array}\right)
\end{aligned}
$$

The proposed CVFEM and its computer implementation were used to solve the proposed test problems with cubical domains and three different uniform grids, with the following number of nodes in the $x, y$, and $z$ directions, arranged in a line-by-line pattern: $6 \times 6 \times 6$ nodes; $11 \times 11 \times 11$ nodes; $21 \times 21 \times 21$ nodes, and $31 \times 31 \times 31$ nodes. In all cases, the overall iterations of the aforementioned SIVA solution procedure were continued until all normalized residues were less than $10^{-12}$.

In all cases, excellent numerical results were obtained. With the three-dimensional linear temperature distribution, Eq. (21), the numerical solutions matched the exact solution perfectly (within round-off precision), for the coarsest to the finest grids. These results were expected, as the proposed CVFEM is formally second-order accurate [Baliga and Patankar (1980); Baliga (1997)] for the solutions of such problems. For the cases with the nonlinear temperature distributions, Eqs. (22) and (23), the numerical results were also excellent, with relative differences between the numerical and exact solutions all less than $10^{-10}$ for the finest grid ( $31 \times 31 \times 31$ nodes).

Details of the numerical results are not given here (either in tabular or graphical forms) as they matched the aforementioned analytical solutions to almost machine precision in all cases. It should also be noted that in the test problems described above, the convergence of the above-mentioned SIVA iterative solution procedure was achieved (with all normalized residues less than $10^{-12}$ ) without any difficulties, even with the relatively simple plane-by-plane-cum-line-by-line technique that was used to solve the sets of linear, or nominally linear, discretized equations in Step 3 of this procedure. No details of the rate of convergence of 
the SIVA procedure are provided here, as the emphasis in this work was on the formulation of the proposed CVFEM, rather than optimization of the solver or enhancements of its rate of convergence using multigrid or bi-conjugate gradient methods [Briggs (1987); Van der Vorst (1992)].

\section{CONCLUSION}

The formulation and testing a control-volume finite element method (CVFEM) for the prediction of three-dimensional, linear and nonlinear, diffusion-type phenomena in anisotropic media in irregular calculation domains were presented and discussed in this paper. The proposed CVFEM adds to the repertoire of numerical methods available in the published literature for the solution of three-dimensional anisotropic diffusion-type problems. It appears to be the first such method in which four-node tetrahedral elements and node-centered (or vertex-based) polyhedral control volumes are used in the formulation. These features make it particularly attractive for the solution of problem with complex geometries, and also future amalgamation with adaptive-grid techniques [Minkowycz et al. (2006)].

The proposed three-dimensional CVFEM and its computer implementation were tested using several steady conduction-type problems, for which analytical solutions were constructed using a special technique proposed by Patankar (1980). In all cases, the agreement between the numerical and analytical solutions was excellent.

\section{ACKNOWLEDGEMENTS}

Financial support of this research work by the Natural Sciences and Engineering Research Council (NSERC) of Canada, in the form individual operating grants awarded to the third author, is gratefully acknowledged. Financial support received by the second author through the MITACS Accelerate program is also gratefully acknowledged, and special thanks are extended to Professor Ferri Hassani for the related arrangements and supervision.

\section{REFERENCES}

Artemov, V., Beale, S.B., deVahl Davis, G., Escudier, M.P., Fueyo, N., Launder, B.E., Leonardi, E., Malin, M.R., Minkowycz, W.J., Patankar, S.V., Pollard, A., Rodi, W., Runchal, A., and Vanka, S.P., A tribute to D.B. Spalding and his contributions in science and engineering, Int. J. Heat Mass Transfer, Vol. 52, pp. 3884-3905, 2009.

Baliga, B.R., Control-volume finite element methods for fluid flow and heat transfer, in Advances in Numerical Heat Transfer, W.J. Minkowycz and E.M. Sparrow (Editors), Vol. 1, Chapter 3, TaylorFrancis, Washington D.C., pp. 97-135, 1997.

Baliga, B.R., and Atabaki, N., Control-volume-based finite difference and finite element methods, in Handbook of Numerical Heat Transfer, $2^{\text {nd }}$ ed., W.J. Minkowycz, E.M. Sparrow, and J.Y. Murthy, (Editors), Chapter 6, John Wiley \& Sons, New York, 2006.

Baliga, B.R., and Patankar, S.V., A new finite-element formulation for convection-diffusion problems, Num. Heat Transfer, Vol.3, pp.393409, 1980.

Bejan, A., Convection Heat Transfer, John Wiley \& Sons, New York, 1984.

Briggs, W.L., A Multigrid Tutorial, SIAM, Philadelphia, Pennsylvania, U.S.A., 1987.
Das, D.B., Nassehi, V., and Wakeman, R.J., A finite volume model for the hydrodynamics of combined free and porous flow in sub-surface regions, J. Adv. Env. Res., Vol.7, pp.35-38, 2002.

Delleur, J., The Handbook of Groundwater Engineering, CRC Press, Boca Raton, Florida, 1999.

Ferguson, W.J., A control-volume finite element numerical simulation of the high temperature drying of spruce, Drying Technology, Vol. 13, pp. 607-634, 1995.

Ferguson, W.J., The control volume finite element numerical solution technique applied to creep in softwoods, Int. J. Solids Structures, Vol.35, pp. 1325-1338, 1998.

Ferguson, W.J., and Turner, I.W., A comparison of the finite element and control volume numerical solution techniques applied to timber drying problems below the boiling point, Int. J. Num. Meth. Eng., Vol.38, pp.451-467, 1995.

Ferguson, W.J., and Turner, I.W., A control volume finite element numerical simulation of the drying of spruce, J. Comp. Physics, Vol.125, pp.59-70, 1996.

Haji-Sheik, A., and Sparrow, E.M., The solution of heat conduction problems by probability methods, ASME J. Heat Transfer, Vol. 89, pp. 121-131, 1967.

Haji-Sheik, A., Monte Carlo methods, in Handbook of Numerical Heat Transfer, W.J. Minkowycz, E.M. Sparrow, G.E. Schneider, and R.H. Pletcher (Eds.), Chapter 16, pp. 673-722, John Wiley \& Sons, New York, 1988.

Harrild, D.M., and Henriquez, C.S., A finite volume model of cardiac propagation, Annals Biomedical Eng., Vol. 25, pp. 315-334, 1997.

Jayantha, P.A., and Turner, I.W., A second order finite volume technique for simulating transport in anisotropic media, Int. J. Num. Meth. Heat and Fluid Flow, Vol. 13, pp. 31-56, 2003a.

Jayantha, P.A., and Turner, I.W., On the use of surface interpolation techniques in generalized finite volume strategies for simulating transport in highly anisotropic porous media, J. Comp. Appl. Math., Vol.15, pp. 199-216, 2003 b.

Jayantha, P.A., and Turner, I.W., A second order control-volume finiteelement least-squares strategy for simulating diffusion in strongly anisotropic media, J. Comp. Appl. Math., Vol.23, pp. 1-16, 2005.

Kaviany, M., Principles of Heat Transfer in Porous Media, SpringerVerlaag, New York, $2^{\text {nd }}$ Edition, 1995.

LeDain-Muir, B., and Baliga, B.R., Solution of three-dimensional convection-diffusion problems using tetrahedral elements and floworiented upwind interpolation functions, Num. Heat Transfer, Vol. 9, pp. 143-162, 1986.

Lyuksyutov, I.F., Everts, H.U., and Pfnur, H., Diffusion in a strongly correlated anisotropic overlayer, J. Surface Science, Vol.481, pp.124134, 2001.

Mikhailov, M.D., and Ozisik, M.N., On general solution of heat conduction in an anisotropic medium, Int. Com. Heat Mass Transfer, Vol.8, pp.329-335, 1981.

Mikhailov, M.D., and Ozisik, M.N., Unified Analysis and Solutions of Heat and Mass Diffusion, John Wiley \& Sons, New York, 1984.

Minkowycz, W.J., Sparrow, E.M., and Murthy, J.Y., Handbook of Numerical Heat Transfer, $2^{\text {nd }}$ Ed., John Wiley \& Sons, New York, 2006.

Murthy, J.Y., and Mathur, S.R., Computation of anisotropic conduction using unstructured meshes, ASME J. Heat Transfer, Vol.120, pp.583591, 1998.

Nield, D.A., and Bejan, A., Convection in Porous Media, $2^{\text {nd }}$ Ed., Springer-Verlaag, New York, 1999.

Oosthuizen, P.H., and Naylor, D., An Introduction to Convective Heat Transfer Analysis, McGraw-Hill, New York, 1999.

Ozisik, M.N., Heat Conduction - Theory and Applications, John Wiley \& Sons, Inc., 1980. 
Ozisik, M.N., and Shouman, S.M., Transient heat conduction in an anisotropic medium in cylindrical coordinates, J. Franklin Institute, Vol.309, pp457-472, 1980.

Ozisik, M.N., Finite Difference Methods in Heat Transfer, CRC Press, Boca Raton, Florida, 1994.

Patankar, S.V., Numerical Heat Transfer and Fluid Flow, Hemisphere, Washington, D.C, 1980.

Perre, P., and Degiovanni, A., Control-volume formulation of simultaneous transfers in anisotropic porous media simulations of softwood drying at low and high temperature, Int. J. HeatMass Transfer, Vol.33, pp.2463-2478, 1990.

Perre, P., and Turner, I.W., A 3-D version of TransPore: a comprehensive heat and mass transfer computational model for simulating the drying process of porous media, Int. J. Heat Mass Transfer, Vol.42, pp.45014521, 1999.

Plumb, O.A., Spolek, G.A., and Olmstead, B.A., Heat and mass transfer in wood during drying, Int. J. Heat Mass Transfer, Vol.28, pp.16691678, 1985.

Poon, K.C., and Chang, Y.P., Transformation of heat conduction problems from anisotropic to isotropic, Int. Com. Heat Mass Transfer, Vol.5, pp.215-221, 1978.

Poon, K.C., Transformation of heat conduction problems in layered composites from anisotropic to orthotropic, Int. Com. Heat Mass Transfer, Vol.6, pp.503-511, 1979.

Rabbani, M.G., Direct formulation finite element (DFFE) method for groundwater flow modeling: two-dimensional case, Soc. Ind. Appl. Math, Vol.54, pp.674-687, 1994.

Reddy, J.N., and Gartling, D.K., The Finite Element Method in Heat Transfer and Fluid Dynamics, CRC Press, Boca Raton, Florida, $2^{\text {nd }}$ Ed., 2001.

Schwartz, F.W., and Zhang, H., Fundamentals of Ground Water, John Wiley \& Sons, New York, 2003.

Sebben, S., and Baliga, B.R., Some Extensions of Tridiagonal and Pentadiagonal Matrix Algorithms, Num. Heat Transfer, Part B, Vol. 28, pp. 323-351, 1995.
Strang, G., and Fix, G.J., Analysis of the Finite Element Method, PrenticeHall, New Jersey, 1973.

Traiano, R.M., Cotta, R.M., and Orlande, H.R.B., Improved approximate formulations for anisotropic heat conduction, Int. Com. HeatMass Transfer, Vol.24, pp.869-878, 1997.

Truscott, S.L., and Turner, I.W., An investigation of the accuracy of the control-volume finite element method based on triangular elements for simulating diffusion in anisotropic media, Num. Heat Transfer, Part B, Vol. 46, pp. 243-268, 2004.

Turner, I.W., and Ferguson, W.J., An unstructured mesh cell-centered control volume method for simulating heat and mass transfer in porous media: application to softwood drying - Part 1: The isotropic model, $J$. Appl. Math. Modeling, Vol. 19, pp.654-667, 1995.

Turner, I.W., and Ferguson, W.J., An unstructured mesh cell-centered control volume method for simulating heat and mass transfer in porous media: application to softwood drying - Part 2: The anisotropic model, J. Appl. Math. Modeling, Vol. 19, pp.669-674, 1995.

Van der Vorst, H.A., Bi-CGSTAB: A fast and smoothly converging variant of Bi-CG for the solution of nonsymmetric linear systems, SIAM J. Scientific and Statistical Computing, Vol. 13, pp. 631-644, 1992.

Versteeg, H.K., and Malalasekera, W., An Introduction to Computational Fluid Dynamics: The Finite Volume Method, Longman Scientific \& Technical, Harlow, Essex, England, 1995.

Whitaker, S., The Method of Volume Averaging, Kluwer Academic, Boston, 1999.

Yang, J.H. and Lee, S.L., Effect of anisotropy on transport phenomena in anisotropic porous media, Int. J. Heat Mass Transfer, Vol.42, pp.26732681, 1999.

Zienkiewicz, O.C., and Taylor, R.L., The Finite Element Method, $5^{\text {th }}$ Ed., Butterworth-Heinemann, Oxford, England, 2000. 